$0-402$

DOES HEAVY PHYSICAL WORKLOAD OR LOW DECISION AUTHORITY AGGRAVATE THE EFFECT OF MUSCULOSKELETAL PAIN ON THE RISK OF POOR SELFREPORTED PHYSICAL WORKABILITY? A COHORT STUDY OF SWEDISH MEN AND WOMEN

${ }^{1}$ Kathryn Badarin, Lena Hillert, Tomas Hemmingsson, Katarina Kjellberg. ${ }^{1}$ Karolinska Insitutet, Sweden

\subsection{6/OEM-2021-EPI.92}

Introduction Musculoskeletal pain (MSP) can affect the functional capacity of workers and engender reduced work ability (WA). The effect of MSP on WA may differ between workers with and without heavy physical workload/low decision authority. Although MSP is widespread in the workforce, only a few studies have explored this hypothesis.

Objectives To investigate the separate and combined effects of MSP and heavy physical workload/low decision authority on poor self-reported physical WA.

Methods This study uses baseline data from the 2010 Stockholm Public Health Cohort (SPHC) questionnaire. The sample included 9419 workers with good baseline physical WA. Exposure to heavy physical workload and low decision authority were estimated using sex-specific job exposure matrices. The mean values for each exposure were dichotomised at the median (high/low) then combined with the presence of MSP. Follow-up data on poor self-reported physical WA were taken from the 2014 SPHC questionnaire. Logistic regression was performed adjusting for age, education, smoking, BMI, health conditions, psychological distress, and leisure-time physical activity. Additive interaction was estimated using the synergy index (SI).

Results MSP, heavy physical workload and low decision authority were separately associated with poor WA. MSP was associated with higher odds of poor WA than heavy physical workload/low decision authority for women, the opposite was observed for men. Combined exposure to MSP and heavy physical workload/low decision authority was associated with the highest odds of poor WA (e.g, MSP and heavy physical workload men: AOR: 4.04, 95\%CI: 2.00-8.15; women: AOR: 3.25 95\%CI: 1.81-5.83). However, the SI was non-statistically significant for both sexes.

Conclusion Combined exposure to MSP and heavy physical workload/low decision authority was associated with higher odds of poor WA than exposure to each factor separately. However, heavy physical workload/low decision authority did not statistically significantly aggravate the effect of MSP on the risk of poor WA.

\section{0-474 PREVALENCE AND RISK FACTORS FOR WORK-RELATED NECK PAIN IN CIVIL SERVANTS: A SYSTEMATIC REVIEW}

${ }^{1}$ Ivan Manuel Nicolau França Chivambo, Fernando Feijó, Paulo Oliveira, Daniela Monteiro. 'Universidade Federal do Rio Grande do Sul, Brazil

\subsection{6/OEM-2021-EPI.93}

Introduction Neck pain is a common work-related musculoskeletal outcome. To summarize its occurrence and risk factors may help to guide occupational interventions, minimizing the problem. Objective To verify the prevalence and risk factors associated with occupational neck pain in employees.

Method This is a systematic review, with a search performed in two databases (PubMed/Medline and BIREME). Publications that estimated the prevalence of occupational neck pain and associated factors among employees were included in the review. Two independent reviewers searched, selected and evaluated all articles based on the PRISMA protocol.

Results 1830 articles were identified, and 48 which met the inclusion criteria were analyzed. Number of participants ranged from 15 to 134754 individuals among the studies; 28 studies (56\%) were cross-sectional, and 23 (44\%) were cohort studies; 74\% came from Asia, Europe and Oceania, 8\% from America, and 6\% from Africa. Most studies analyzed musculoskeletal disorders in general, and neck pain was the second most prevalent complaint. The highest prevalence of neck pain was 71\%, and the outcome was more frequent in women. The main risk factors described were ergonomic, psychological and social aspects.

Conclusions Neck pain is the second most prevalent musculoskeletal disorder in civil servants, particularly in office workers using computers. The most common associated individual risk factors were female gender, age, body mass index, and previous history of neck pain. Ergonomic risk factors were time and frequency of use of the mouse and keyboard, the position and height of the monitor, the temperature in the work environment, the posture adopted in the chair, as well as the simultaneous use of the telephone and computer.

\section{0-484 OCCUPATIONAL LIFTING, PUSHING AND RISK OF SUBACROMIAL IMPINGEMENT SYNDROME SURGERY: A QUANTITATIVE RISK ASSESSMENT}

${ }^{1}$ Annett Dalbøge, Johan Hviid Andersen, Bjørn Riddervold. 'Department of Occupational Medicine, Denmark

\subsection{6/OEM-2021-EPI.94}

Objective The aim was to examine exposure-response relations between intensities of pushing/pulling, lifting/carrying loads and surgery for subacromial impingement syndrome (SIS) during a 10-year period.

Methods We conducted a register-based cohort study (20032008), comprising all persons born in Denmark (19331977) with at least 5 years of work experience $(\mathrm{N}=2374$ 403). Information on SIS surgery was retrieved from the Danish National Patient Register ( $\mathrm{N}=14$ 188). Occupational mechanical exposures comprising lifting or carrying loads $\geq 10 \mathrm{~kg}$ and pushing or pulling loads $\geq 50 \mathrm{~kg}$ were assessed by combining individual job codes with our expert-based Shoulder job exposure matrix. We created 3 intensity-specific exposure duration variables by dividing the intensity for lifting/carrying and pushing/pulling into categories (low, medium, and high), and summed up the number of years in each exposure category for a 10-year time window. The association was analyzed using logistic regression as survival analysis.

Results The adjusted odds ratio (ORadj) increased with both exposure duration and intensity of lifting/carrying and pushing/pulling. ORadj reached a maximum of $1.78 \quad(95 \%$ CI 1.66-1.89), 2.52 (95\% CI 2.32-2.74), and 2.96 (95\% CI 2.53-3.47) after 10 years of exposures for the three exposure intensities. For pushing/pulling, maximum ORadj was 1.44 (95\% CI 1.31-1.58), 1.68 (95\% CI 1.58-1.79), and 1.72 (95\% CI 1.50-2.00), respectively.

Conclusion We found exposure-response relations for both lifting/carrying and pushing/pulling across the 10-year time 